Cristea I. A., Gentili C., Cotet C. D., et al (2017) Efficacy of psychotherapies for borderline personality disorder. a systematic review and meta-analysis. JAMA Psychiatry, 74(4), 319-28.

Fonagy P., Luyten P., Moulton-Perkins A., et al (2016) Development and validation of a self-report measure of mentalizing: the reflective functioning questionnaire. PLOS ONE, 11(7), e0158678.

Fonagy P., Luyten P. \& Bateman A. (2017) Treating borderline personality disorder with psychotherapy: where do we go from here? JAMA Psychiatry, 74(4), 316-7.

Gunderson J. (2014) Handbook of Good Psychiatric Management for Borderline Personality Disorder. American Psychiatric Publishing.

Hersh R. G., Caligor E. \& Yeomans F. E. (2017) Fundamentals of Transference-Focused Psychotherapy: Applications in Psychiatric and Medical Settings. Springer.
Hörz-Sagstetter S., Caligor E., Preti E., et al (2017) Clinician-guided assessment of personality using the Structural Interview and the Structured Interview of Personality Organization (STIPO). Journal of Personality Assessment, doi: 10.1080/00223891.2017.1298115.

Kernberg O. F. (2015) Resistances and progress in developing a research framework in psychoanalytic institutions. Psychoanalytic Inquiry, 355, 98-114.

Paris J. (2015) Concise Guide to Personality Disorder. American Psychological Association.

Stoffers J. M., Völlm B. A., Rücker G., et al (2012) Psychological therapies for people with borderline personality disorder. Cochrane Database of Systematic Reviews, 15(8), CD005652.

\title{
COUNTRY \\ Mental healthcare in Saint Lucia
} PROFILE

\author{
Karen A. Francis, ${ }^{1}$ Andrew Molodynski, ${ }^{2}$ and Giselle Emmanuel ${ }^{3}$
}

${ }^{1}$ Senior Nursing Lecturer, Spartan Health Sciences University, Saint Lucia;

email karenf76@hotmail.com

${ }^{2}$ Consultant Psychiatrist, Oxford Health National Health Service Foundation Trust; Honorary Senior Lecturer, Oxford University, UK

${ }^{3}$ Charge Nurse, Saint Lucia National Mental Wellness Centre Saint Lucia

\section{Conflicts of interest. None.}

(c) The Authors 2018. This is an Open Access article, distributed under the terms of the Creative Coper the terms of the Commons Attribution-

NonCommercial-NoDerivatives licence (http://creativecommons. org/licenses/by-nc-nd/4.0/), which permits non-commercial re-use, distribution, and reproduction in any medium, provided the orany medium, provided the original work is unaltered and is properly cited. The written permission of Cambridge University Press must be obtained for commercial re-use or in order to create a derivative work.

\begin{abstract}
St Lucia is a small island in the eastern Caribbean with a population of approximately 200000 people. Although St Lucia is formally ranked as a high middle-income country, there are pockets of deprivation and relatively low living standards. Mental health services in St Lucia have increased considerably and advanced over recent years because of a coalition between the government of the island and South East Asian partners. The National Mental Wellness Centre opened several years ago and has much improved facilities. There remains a significant shortage of community-based services, no mental health law, and a pervasive community stigma and apprehension regarding those with mental health problems.
\end{abstract}

In St Lucia there is a relative lack of data regarding the provision and outcomes of mental health services. St Lucia is an island state in the eastern Caribbean with a population of 200000 . It is classified as an upper middle-income country by The World Bank (2012). Despite this, there are pockets of deprivation and many people live under the poverty line. An institution previously known as 'Golden Hope Hospital' was the first step towards mental healthcare on the island. The hospital was run as a custodial asylum, with a strong medical focus and little input from other professionals. Although designed as a 'place of refuge,' in reality it was more about the containment of some of the island's most vulnerable people. It was replaced in 2010 by the National Mental Wellness Centre in a collaborative effort between the governments of St Lucia, China and Taiwan. The centre provides a multidisciplinary model of care with doctors, nurses, psychologists, occupational therapists and social workers collaborating closely. Services (including medications) are available free of charge to individuals regardless of age, nationality and economic status (WHO, 2009).

\section{Structure and demographics}

National health expenditure is $8 \%$ of the gross domestic product and $4 \%$ of that is allocated to mental healthcare, of which $97 \%$ goes to mental hospitals (WHO, 2011). There is no separate budget for community-based mental health services, and what exists is financed from the hospital budget. As a result, limited funds are available to strengthen the delivery of primary care services for people with mental disorders. The majority of hospitalised patients have a diagnosis of schizophrenia, other psychosis or substance-induced psychosis, but good quality data are hard to find (WHO, 2009). There are 71.8 beds per 100000 people, none of which are specifically designated for children and adolescents. These beds are all at the wellness centre where there are two acute wards (one male and one female), three rehabilitation wards, and a drug rehabilitation and counselling service, known as Turning Point.

Two psychiatric consultants and two registrars take charge of the day-to-day care of patients. There is one psychotherapist and one social 
worker. There is also an occupational therapy department that is staffed by an occupational therapist, three rehabilitation assistants and one craft assistant. The nursing team consists of registered nurses, registered nursing assistants, health aids and orderlies; none are formally trained in mental health. There is one mental health-trained nursing practitioner, who acts as the principal nursing officer. There is one social worker for the entire psychiatric department, which is inadequate to provide an efficient and effective service. There is also one clinical psychologist post which is currently vacant.

There is no forensic mental healthcare. Prisoners with mental disorders are treated and monitored in the prison by staff from the mental hospital. They can also access the mental health services at out-patient clinics under close supervision of prison officers.

Aside from the hospital, mental health services are offered at 9 of the island's 34 health centres. Currently, there are three psychiatrically trained nurse practitioners who carry out communitybased mental healthcare in monthly scheduled clinics in the nine health centres. There are no day treatment facilities or community-based psychiatric in-patient units. The best estimates for 2007 indicated that approximately 100 patients were treated in primary healthcare settings. Again, high quality data are lacking (WHO, 2009). When space is limited, general acute hospitals admit patients with acute psychiatric illnesses to medical wards. In the general hospital, care is given based on the Emergency Severity Index. People are thus categorised based on presentation.

\section{Policy and legislation}

There is currently no mental health authority in St Lucia (WHO, 2011). However, within the Ministry of Health there is a mental health reform project for developing a comprehensive mental healthcare system. Although the government drafted a Mental Health Policy in 2007, a revised mental health strategic plan in 2007/08-2010/11 and a draft Mental Health Act in 2008, none have yet come into force.

In their review of the Commonwealth member states' mental health legislation, Pathare et al (2014) highlighted the fact that no mental health law, human rights policy or review body exists in St Lucia. There is, however, a Disaster Emergency Plan for Mental Health, which has been in place since 2000 (PAHO, 2012). There is no national coordination of public education or awareness campaigns on mental health-related issues (Pathare et al, 2014). There is also limited access to social support systems for the mentally ill as the national insurance payment scheme does not cover most people with mental health problems. However, social welfare benefits are available to people on the basis of disability due to mental disorders (WHO, 2009).

\section{Challenges}

Although the National Mental Wellness Centre has been a real step forwards in the past decade, many challenges in St Lucia persist.

\section{Shortage of hospital beds and lack of alternatives}

An increasing number of admissions (particularly of people with substance misuse) and limited bed spaces is compounded by many patients being abandoned, and thus left at the National Mental Wellness Centre long term, even after their treatment regime has been completed (SNO, 2015).

Services do not currently provide a comprehensive range of therapeutic or rehabilitative interventions. There is a strong focus on medication (which of course is important), without other forms of rehabilitation and support. It is well established that a multidimensional care approach is more effective in practice (Burns, 2004), particularly when objectives are focused, quantifiable and reproducible (Machado-Vieira et al, 2004). Lack of investment has hindered the development of such approaches.

\section{Stigma and discrimination}

Negative stereotypes and unfounded beliefs within the general public often lead to ostracism and, in some cases, abuse of the mentally ill. The executive director of the National Mental Wellness Centre highlighted this as a potential reason for the abandonment of patients in the hospital (SNO, 2015). As is the case elsewhere, this can be compounded by negative staff attitudes and stigma.

\section{Staff training and supervision}

Reports have highlighted major inadequacies in staff training and supervision (WHO, 2009). There is also a lack of external scrutiny of services, with no one trained to do this. Many nurses are inexperienced and acquired the role by default, straight after nursing school. Even experienced staff may fail to follow recognised care pathways.

\section{Legislation and policy}

As described above, the absence of a clearly defined mental health policy for St Lucia and the absence of mental health legislation are areas of significant concern still to be remedied.

\section{Reasons for non-implementation of structures and policies}

Two major reviews of the English-speaking Caribbean (Saxena et al, 2006; Caldas de Almeida, 2013) suggested that the development and implementation of mental health policies and services is hindered by the small size and limited capacity of many states.

\section{Positive initiatives and future directions}

The occupational therapy department at the National Mental Wellness Centre is providing 
some psychosocial forms of treatment with an emphasis on recovery. The St. Lucia Times (2016) highlighted the department's recent efforts towards including the mental health patients in the island's jazz festival by organising a jazz session on the hospital grounds. The department also engages clients in projects such as gardening and crafts, from which products are sold and the proceeds are used to support the running of the department. This helps build self-esteem and enables the acquisition of skills that the person can use to support themselves after being discharged.

The long-term aim is to combine three institutions (National Mental Wellness Centre, Turning Point and Owen King EU Hospital) into a mental health complex to increase community-based care and subsequently reduce admissions. The inclusion of a child and adolescent ward will form part of the changes.

\section{Recommendations and conclusions}

Mental healthcare in St Lucia has a number of challenges such as underinvestment, stigma, policy and legal gaps, and a lack of community services. There have been positive steps over recent years, however, the limited evidence available locally and information from other sources suggest four major areas in which improvement is needed.

First, a cross-sectoral approach with formal and informal links to a wide range of agencies needs to be in place to mobilise support for mental health activities. Mental health practitioners, including trained doctors, need to be involved at all levels of governmental institutions.

Second, training sessions covering priority areas, such as the organisation of services, legislation and human rights, and mental health promotion and prevention, need to be set up within all sectors as well as within communities. This should reduce stigma, increase awareness and increase competencies in a wide range of mental health-related areas.

Third, a wide range of therapeutic options, such as day hospitals, group therapy, individual case management and easy access to health services, should be made available to patients and their families, especially within primary care settings. Collaborative multidisciplinary working and crisis support, specifically in terms of case allocation and care management, should improve prognosis and increase the flexibility of response to need.
Fourth, the adoption of mental health legislation and policy would help create unified standards of care, treatment pathways and human rights. Organised mental health data, recorded in a national health information system, is crucial to monitor progress or difficulties in the system.

These changes are crucial to build on the positive advances of recent years and deliver a good quality, reliable and comprehensive system of mental healthcare to the population of St Lucia.

\section{References}

Burns T. (2004) Community Mental Health Teams: A Guide to Current Practices. Oxford University Press.

Caldas de Almeida J. M. (2013) Mental health services development in Latin America and the Caribbean: achievements, barriers and facilitating factors. International Health, 5, 15-18.

Machado-Vieira R., Santin A. \& Soares J. C. (2004) O papel da equipe multidisciplinar no manejo

do paciente bipolar. [The multidisciplinary team approach to the treatment of bipolar disorder: an overview]. Rev Bras Psiquiatr., 26 Suppl 3, 51-53. Available at http://www.scielo.br/scielo.php?pid=S151644462004000700012\&script=sci_arttext\&tlng=en on 9/9/2016.

Pan American Health Organization (PAHO) (2012) Saint Lucia. In Health in the Americas (Country Profiles Edition): 562-76.

Pathare S., Shields L. \& Nardodkar R. (2014) A Review of Mental Health Legislation in Commonwealth Member States. Commonwealth Health Partnerships. Available at http://www.commonwealthhealth. org/wp-content/uploads/2014/05/3-mental-health-legislationpathare.pdf (accessed 13 September 2016).

Saint Lucia News Online (SNO) (2015) Mental Wellness Centre being used as "dumping ground" - official. 13 February. Available at http:// www.stlucianewsonline.com/mental-wellness-centre-being-used-asdumping-ground-official/ (accessed 1 August 2016).

St. Lucia Times (2016) Music Therapy at Mental Wellness Centre. 17 May. Available at http://stluciatimes.com/2016/05/17/musicaltherapy-mental-wellness-centre (accessed 1 September 2016).

Saxena S., Pratap S., Garrido M., et al (2006) World Health Organization's mental health atlas 2005: implications for policy development. World Psychiatry, 5, 179-84.

The World Bank (2012) Saint Lucia - Multiple Indicator Cluster Survey. Available at http://data.worldbank.org/country/st-lucia (accessed 29 September 2016).

World Health Organization (WHO) (2009) World Health Organization Assessment Instrument for Mental Health Systems (WHO-AIMS) in Saint Lucia (Internet). World Health Organization. Available at http://www.who.int/mental_health/who_aims_report saint_lucia.pdf (accessed 1 August 2016).

World Health Organization (WHO) (2011) Saint Lucia, Mental Health Atlas. Department of Mental Health and Substance Abuse, World Health Organization. Available at http://www.who.int/mental_health/ evidence/atlas/profiles/lca_mh_profile.pdf?ua=1 (accessed 29 September 2016). 